\title{
Control of vergence:
}

\section{Gating among disparity inputs by voluntary target selection}

\author{
C.J. Erkelens* and H. Collewijn \\ Department of Physiology I, Faculty of Medicine, Erasmus University, P.O. Box 1738, 3000 DR Rotterdam, The Netherlands
}

Received February 22, 1991 / Accepted April 19, 1991

Summary. The ability of human beings to shift their vergence between multiple disparity stimuli was investigated. The stimulus was a stereogram consisting of a single bar $\left(1 \times 0.3^{\circ}\right)$ projected in the center of a larger circular pattern $\left(28^{\circ}\right.$ dia) of dots $\left(0.3^{\circ}\right.$ dia $)$. In the initial condition, the subjects perceived a single bar in the center of a cluster of dots, all lying in a single depth plane. The subjects were instructed to fixate either the bar or one of the dots close to the bar. Stepwise changes of target-vergence of either the bar, or of all dots, or of both configurations in opposite directions, were imposed in a random sequence. Under these conditions, ocular vergence was controlled by the target-vergence of the selected target at all times, even if this implied a loss of binocular fusion for the non-selected target. The effect of target selection per se, without concomitant changes in retinal position of the selected target, was studied in two experienced subjects with stabilized image techniques. The subjects viewed a configuration containing short vertical bars placed at either side of a long vertical bar. The short bars on different sides of the long bar had opposite (crossed or uncrossed) disparities. After stabilization of the configuration while the subject fixated the long bar, subjects attempted to fixate the short bars alternatingly. Due to the stabilization the ensuing eye movements did not affect the retinal positions, and thus the disparity, of the bars. Attempted fixation of a parafoveally viewed, short bar induced vergence responses in the appropriate direction. These vergence responses would have reduced disparity of the selected target if this had not been stabilized, at the expense of increasing disparity of non-selected targets. From this result we conclude that, by the mechanism of target selection, disparity information of the selected target is gated to a subsystem processing disparity which controls vergence.

\footnotetext{
* Present address: Department of Medical and Physiological Physics, Buys Ballot Laboratory, University of Utrecht, P.O. Box 80000 , 3508 TA Utrecht, The Netheriands

Offprint requests to: C.J. Erkelens
}

Key words: Binocular eye movements - Ocular vergence Target selection - Human

\section{Introduction}

A stimulus in one retina can be physiologically fused with a range of stimuli around the point of precise correspondence in the other retina. Vergence movements provide the conditions under which the sensory process of fusion can successfully operate to obtain or maintain a single percept. It is generally accepted that vergence movements are dominated by foveal stimulation. Peripheral stimuli, however, have been shown to contribute significantly too. Burian (1939) found that peripheral fusion could disrupt central fusion, causing the subject to experience diplopia in the perception of a small fusional stimulus within the central visual field. Hampton and Kertesz (1982), who measured binocular eye movements objectively, found that small peripheral stimuli in the absence of foveal stimuli induced adequate vergence responses. Regan et al. (1986) found similar results for the peripheral areas where the subjects perceived stereomotion. Kertesz (1981) investigated the effect of size and complexity of the stimulus on vergence responses. There was an almost threefold increase in the vergence amplitude as the size of the single line stimulus increased from $5^{\circ}$ to $57.6^{\circ}$. Erkelens (1987) compared vergence speeds induced by a single-line stimulus $\left(10 \times 0.5^{\circ}\right)$ with those induced by a random-dot configuration $\left(12 \times 12^{\circ}\right)$ and found that the random-dot stimulus was more effective.

In the literature cited here, foveal stimuli were either absent or had disparities of similar polarity, crossed or uncrossed, as the disparities of the peripheral stimuli. Under such conditions, peripheral stimuli contribute positively to vergence responses in that they enhance binocular foveation. This condition will generally not exist during viewing of normal scenes in which multiple disparity stimuli are present. Appropriate binocular fixation of one 
target will automatically imply that other stimuli lying on different depth surfaces are projected on non-corresponding retinal positions. Such stimuli may even be located in the foveae. In view of the experiments mentioned before, foveal and peripheral disparity stimuli, which are not meant to be fixated, may hamper or even prevent the binocular fixation of a specific target. This conclusion seems to be at odds with experience in viewing under normal conditions. In natural stimulus conditions, human beings can easily change their binocular fixation from one object to another visible object lying on a different depth surface (Erkelens et al. 1989b). One could argue that under normal viewing conditions objects lying on different depth surfaces will be blurred and, therefore, lose their power as a stimulus for disparity-induced vergence. An alternative explanation may be that human beings can select particular stimuli and disregard others for driving vergence. The present experiments were carried out to investigate this hypothesis. Therefore, stimuli were presented which contained foveal and peripheral disparity stimuli, but which allowed the subject to follow or to ignore imposed disparity changes. In a first experiment, changes in target vergence were imposed either on the center or on the periphery of a large stereogram, while subjects were asked to attend either to the central or the peripheral part.

The rationale for presenting such disparity stimuli was that the dynamics of the resulting vergence responses, which might be gated by the voluntary selection, could be compared to the dynamics of disparity vergence responses described in the literature. The experiments demonstrated that vergence was controlled by the part of the stereogram that was voluntarily attended, rather than by the part of the stereogram that was projected onto the foveae. As in this experiment shifts in the attended target were still accompanied by shifts in the retinal image position, a second experiment was designed in which retinal image shifts were excluded by a stabilization technique. This experiment demonstrated conclusively that vergence was driven by that part of a (stable) retinal image configuration which was selectively attended to. Thus, gating of vergence control between multiple targets with different disparities can be effected by selective attention alone, without any change in the retinal image conditions such as position or blur.

Prelimary results of these experiments have been reported (Erkelens 1988).

\section{Methods}

\section{Subjects}

Five subjects participated in the experiments. They had visual acuities of 20/20 or better, with (four subjects) or without (one subject) correction. None of them showed any ocular or oculomotor pathologies. Three subjects were experienced in oculomotor research. The two other subjects served in such experiments for the first time. All five subjects served in Experiment I, while two of them, experienced in viewing of stereograms under stabilized conditions, also served in Experiment II. Informed consent was obtained from all subjects prior to undertaking the study.

\section{Apparatus}

Horizontal and vertical eye movements of both eyes were measured with induction coils mounted in scleral annuli in an a.c. magnetic field as first described by Robinson (1963) and modified and refined by Collewijn, Van der Mark and Jansen (1975). The dynamic range of the analog part of the recording system was d.c. to better than $100 \mathrm{~Hz}$ ( $3 \mathrm{~dB}$ down), noise level less than $\pm 1^{\prime}$ and deviation from linearity less than $0.5 \%$ over a range of $\pm 25^{\circ}$. Head movements were minimized by supports under the chin and around the skull.

The stimulus patterns were back-projected onto a large translucent screen $\left(92 \times 82^{\circ}\right)$ at a distance of $143 \mathrm{~cm}$ in front of the subject. $\mathrm{Up}$ to 4 separate images were projected simultaneously. The horizontal position of each image was independently controlled by means of mirrors mounted on galvanometer motors (Model G300 PD General Scanning, Watertown, MA). To separate the left and right half-images of the stereograms, subjects wore a green filter in front of the left eye, and a red filter in front of the right eye (Cinemoid, primary green and primary red). Similar red and green filters were mounted in front of the appropriate projectors. Light separation between the red and green images was better than $99 \%$. The luminance of the images was adjusted to equal brightness for each of the subject's eyes. Luminance was about $14 \mathrm{~cd} / \mathrm{m}^{2}$ for light target elements and $1 \mathrm{~cd} / \mathrm{m}^{2}$ for dark target elements. Target-vergence (Rashbass 1981) of the presented images was defined as the angle subtended between the lines passing through identical features of each half-image and the point of rotation of the eye by which it was viewed. The horizontal position of each image was signalled by a transducer, incorporated into the scanner, and recorded together with the eye movements.

A minicomputer (PDP 11/73), used for stimulus generation, data collection and data analysis, controlled the horizontal movements of the half-images independently, by rotating the servo-controlled mirrors. The images were viewed under stabilized conditions in Experiment II. Retinal image stabilization was achieved for each of the two eyes by using the position signal of each individual eye to drive the mirror deflecting the half-image viewed by that eye, with unity gain. In order to minimize perceptual fading, the images were only stabilized for horizontal movements.

\section{Procedure}

The sensitivity of the eye movement recorder was adjusted at the start of each experimental session. A calibration target containing 9 fixation marks, forming a $3 \times 3$ orthogonal grid, spaced at $10^{\circ}$ intervals, was presented. The subject fixated, monocularly, on each mark in turn while the gain and the offset of the eye position signal were adjusted. After these adjustments, voluntary gaze shifts between the calibration marks were recorded for each eye separately. The calibration recording was repeated at the end of each session.

\section{Experiment $I$}

Subjects had to fixate one part of a stereogram consisting of a center and a background (Fig. 1A). The background of the stereogram was a circular pattern of $28^{\circ}$ diameter which contained about 300 randomly arranged dots, each of $0.3^{\circ}$ diameter.

The center of the stereogram was formed by a single, vertical bar $\left(1 \times 0.3^{\circ}\right)$. In the initial condition the two parts of the stereogram were projected with zero relative disparity between them. The targetvergence of both the bar and the dots was about $2^{\circ}$. The exact angles were slightly different for each subject, depending on his or her interocular distance. While viewing through the red-green goggles the subjects perceived a white bar and a collection of white dots, all lying in the same depth plane.

In the literature dealing with disparity vergence mainly two types of stimuli are used, namely stepwise and sinusoidal changes in disparity. The same type of stimuli were chosen for the present 


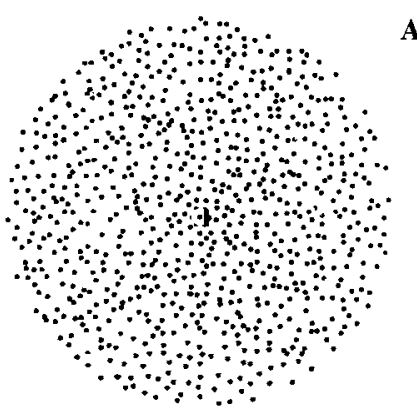

$\mathbf{A}$


Fig. 1A, B. Stimuli used in Experiment I (A), and in Experiment II (B)

experiment in order to enable the comparison of the present results to data in the literature. Stepwise changes in target-vergence of either the bar, or of all dots, or of both configurations in opposite directions, were imposed in a random sequence. Each sequence contained 18 trials which had a duration of $16 \mathrm{~s}$ each. The steps had a period of $4 \mathrm{~s}$. The size of the steps was $2^{\circ}$ in the convergent direction when only the bar was moved, $2^{\circ}$ in the divergent direction when only the dots were moved, and $1^{\circ}$ for each of the parts when both of them were moved simultaneously. The result of this procedure was that the bar had either a crossed disparity of $2^{\circ}$ or zero disparity relative to the dots under the 3 different conditions. In a first series of trials, subjects were instructed to fixate the vertical bar at all times. In a second series of trials, the whole sequence was repeated with the instruction to fixate, at all times, one single dot of the large dot configuration, which was closely adjacent to the vertical bar (the distance was about $0.3^{\circ}$ ). Thus, the whole experiment consisted of 36 trials for each subject.

Sinusoidal changes in target-vergence were presented with frequencies and amplitudes in a range usually used in disparity vergence experiments. The combinations of amplitude and frequency were presented in a random order. The target-vergence of the vertical bar was changed at 5 frequencies ranging from 0.125 to $1.5 \mathrm{~Hz}$, and peakto-peak amplitudes of 1,3 , or $5^{\circ}$. Each combination of amplitude and frequency was presented twice during periods of $32 \mathrm{~s}$. In half of the trials the bar was the only visible stimulus, just as in traditional experiments, while the large dot configuration served as a stationary background in the other half of the trials. The target-vergence of the dots was fixed at $4^{\circ}$ convergence. The movements of the bar were centered around the same angle of convergence. Consequently, the bar was seen at distances closer and further away than the dotbackground during different phases of the sinusoidal movements, without requiring divergent angles between the eyes in the case of accurate tracking.

\section{Experiment II}

In this experiment we examined whether the weight of a specific disparity stimulus in driving vergence was changed just as a result of the fact that this stimulus was selected for binocular fixation. Under normal viewing conditions target selection is associated with eye movements, which change the retinal position of the stimulus, and implicitly its contribution to vergence. The technique of retinal image stabilization allows the subject to select a different target without a concomitant shift of the retinal image.

The stimulus consisted of the 2 fusible half-images shown in Fig. 1B. The left eye viewed an upright cross (bars $40 \times 0.5^{\circ}$ ) on a dark background. The vertical cross-bar was flanked by 4 vertical bars $(15$ $\times 0.5^{\circ}$ ), each placed in one quadrant of the cross at $5^{\circ}$ from the horizontal cross-bar. The vertical bars were placed at a distance of $1^{\circ}$ from the vertical cross-bar in the upper quadrants. The right eye viewed an image which was identical, but rotated by $180^{\circ}$. In the right half-image the vertical bars were placed at a distance of $0.5^{\circ}$ from the vertical cross-bar in the upper quadrants. With binocular viewing through the red-green goggles the subject perceived a stereoscopic image of a single, upright cross, together with 4 vertical bars, each of which was located in a different quadrant. The bars in uneven (upper right and lower left) quadrants appeared to stand in front of the central cross, while the other bars were seen behind the cross.

Subjects, especially when they are wearing scleral contact lenses, find it uncomfortable to view in an upward direction. For that reason, the middle of the upper quadrants was placed at eye level. This position of the stimulus configuration relative to the subject implied that the relative disparity between the two small bars in the upper quadrants was $1^{\circ}$, whereas this disparity was slightly smaller between the bars in the lower quadrants. The retinal images were stabilized in the horizontal direction for each of the eyes separately. Stabilization for only horizontal eye movements allowed free scanning of the images in the vertical direction. Accurate positioning of the stereogram and accurate stabilization allowed free scanning of a fixated, vertical bar without inducing horizontal eye movements. At the beginning of the experiment the quality of image stabilization was checked for each eye separately while only one half-image was presented. In case the eyes and image started to drift horizontally during the scanning of the vertical cross-bar, the calibration procedure was repeated. Only if the image did not drift during vertical scanning at several horizontal eye positions, the quality of image stabilization was considered good enough to carry on with the experiment. Both half-images were then presented and stabilized simultaneously. The subject was first asked to scan the fixated vertical cross-bar. During scanning we verified that the vergence angle between the eyes remained stable. Subsequently the subject was asked to fixate the vertical cross-bar in the upper quadrant, and then to attend to the right flanking bar, and subsequently to the left bar and back and so on. These shifts in attention between the flanking bars were made in a comfortable rhythm of about once each $2 \mathrm{~s}$. The shift in attention were made on command of the experimenter who gave his instructions verbally and who simultaneously recorded his instructions. A sequence of these instruction is shown in the bottom of Fig. 5. By following this procedure, the subject could fully devote his attention to the task and the experimenter could keep the target within a distance of about $8^{\circ}$ from the straight-ahead position in which the quality of stabilization was optimal. A consequence of this procedure was that for the interpretation of the results we had to rely on the assurance given by the subjects to follow the instructions promptly. After recording of the movements of the eyes and the images for a period of $32 \mathrm{~s}$ the experiment was repeated while the vertical cross-bar in the lower quadrant was fixated. The disparities of the flanking bars in the lower quadrant were opposite to those of the bars in the upper quadrant. 
This stimulus configuration enabled us to verify that the occurring changes in vergence were truly induced by the shifts in attention, and not by inaccurate stabilization or unbalance of versional eye movements. The entire experiment was replicated on a different day for each of the two subjects serving in this experiment.

\section{Data analysis}

Horizontal and vertical eye position signals and horizontal mirror positions were digitized on-line at a frequency of $125 \mathrm{~Hz}$ (resolution $0.8^{\prime}, 8 \mathrm{~ms}$ ) after low-pass filtering with a cut-off frequency of $62.5 \mathrm{~Hz}$, and then stored on disk. In the off-line analysis, eye position signals recorded during the calibration trials were first checked for errors in gain and offset between target and eye. An experiment was discarded if the discrepancies exceeded $1 \%$ for gain and $0.2^{\circ}$ for offset.

Ocular vergence was calculated by subtracting the right horizontal eye position from the left horizontal eye position. Target-vergence was calculated by subtracting the position of the mirror deflecting the image seen by the right eye from the position of the mirror deflecting the image seen by the left eye. The vergence error (i.e. absolute retinal disparity) was calculated by subtracting the ocular vergence from the target-vergence. Under stabilized conditions the vergence error had to be very close to zero. Thus, the vergence error signal provided an off-line check on the quality of the stabilization. Generally, vergence errors were smaller than $0.1^{\circ}$ with shortduring excursions during blinks.

For the sinusoidal stimulus movements, the signals of ocular vergence and target-vergence were Fourier transformed with a Fast Fourier Transform routine. Gain (ratio of peak-to-peak amplitudes) and phase (in degrees) between the fundamental components of movements of ocular vergence and target-vergence were calculated by means of auto- and cross-power spectral densities.

\section{Results}

Experiment I: selective vergence tracking of non-stabilized targets

Responses to steps in target vergence. Vergence responses to stepwise changes in disparity, representative for the five subjects, are shown in Fig. 2. The subjects were very successful in minimizing the vergence error of the selected target at the expense of that of the non-selected target. When one of the dots was fixated, ocular vergence followed target-vergence accurately irrespective whether the selected or non-selected target was moved, or both targets were moved in opposite direction. When the bar was fixated, vergence tracking of the selected target was as accurate as when one of the dots was fixated, except in the condition that the bar remained stationary and all the dots were moved. Occasionally, and only in two subjects, ocular vergence transiently followed the target-vergence of the non-selected dot configuration (Fig. 2 left row, middle figure) in this condition. With practice, these incorrect movements grew smaller and ultimately disappeared. The fixation disparity was assessed during a period of one second starting from two seconds after each stimulus jump. The fixation disparity of the selected target was smaller than $0.3^{\circ}$ for all subjects in all trials. Consequently, the disparity of the non-selected target was larger than $1.7^{\circ}$ whenever the selected and non-selected targets were placed in different depth planes. The latency of the ocular
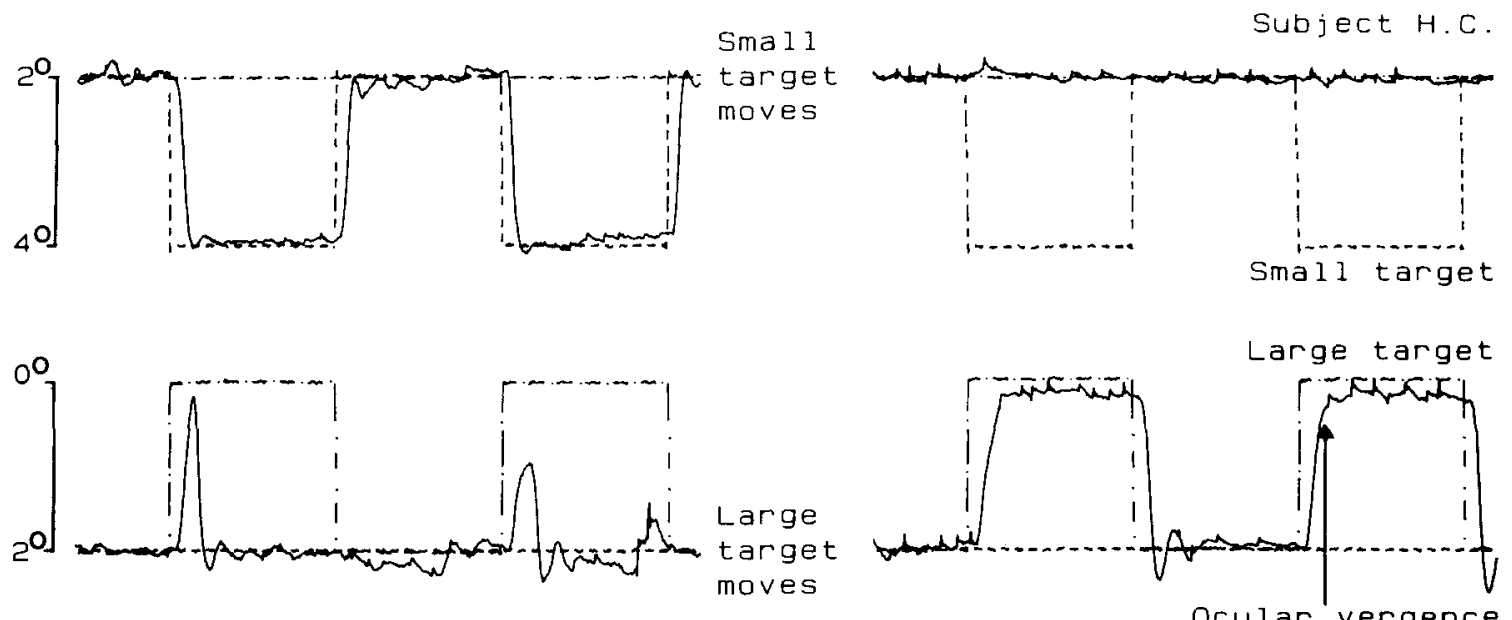

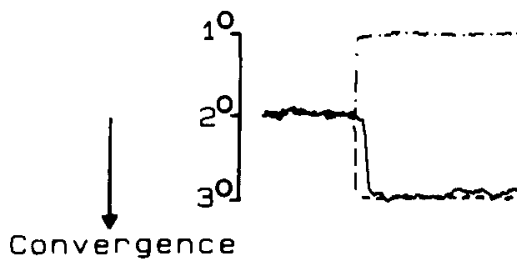

Fig. 2. Representative vergence responses (subject H.C.) to stepwise changes in target-vergence of either the bar (top panels), or the large dot-configuration (middle panels), or both parts of the stereogram simultaneously in opposite directions (bottom panels). The subject was instructed to fixate the central bar (left panels) or a particular dot of the dot-configuration (right panels) 
vergence responses, averaged over the subjects, was 194 $\pm 51 \mathrm{~ms}$. Thus, the subjects were able to follow the changes in target-vergence of the selected part of the stereogram accurately and promptly.

The relative disparity between the bar and the dots changed similarly in all 3 stimulus conditions in which either one of the parts of the stereogram jumped, or both parts jumped in opposite directions. The visual perception of the induced stepwise changes in disparity, however, was very different. When only the small bar jumped from zero disparity to $2^{\circ}$ of crossed disparity, this target became initially unfused while the dot pattern remained fused. When only the large dot pattern jumped from zero disparity to $2^{\circ}$ of uncrossed disparity just the opposite occurred: initially the dots were not fused and the small bar remained fused. When both parts jumped in opposite directions, both of them were transiently not fused. Thus only the part of the stereogram, either the small or large one, which remained projected on corresponding retinal areas was perceived as a binocularly fused, white image, whereas the other part was perceived as non-fused, red-green images. How the perception of the stereogram further developed after the disparity jumps depended on which part of the stereogram was selected as a target. It is important to note that ocular vergence could track the target-vergence of an unfused small stimulus even in the presence of a large fused stimulus.

Responses to sinusoidal changes in target vergence. Subjects were instructed to follow the sinusoidal changes in targetvergence of the single bar, which was presented either alone or together with the stationary large dot configuration. Gain-phase relationships between ocular vergence and target-vergence, averaged over the five subjects, are shown in Fig. 3. An analysis of variance was carried out on the data in which subject, target amplitude and background were the independent variables. The presence of the large, stationary background had only minor effects on the frequency-response of ocular vergence. Phase lags of ocular vergence were similar in both stimulus conditions. They only seemed to differ slightly for the smallest stimulus amplitude $\left(1^{\circ}\right.$ peak-to-peak) tested. However, this difference was not significant. Gains of ocular vergence were also unaffected by a stationary background, except in stimulus conditions with combinations of low frequencies and amplitudes of $3^{\circ}$ and $5^{\circ}$ peak-to-peak. In this case, gain of vergence was slightly lower in the presence of the stationary background. These differences were significant $(p<0.01)$. The main effects of subjects were significant $(p<0.01)$ too. Inspection of the individual ocular vergence recordings showed that in one subject vergence tended to drift away from the small target. Such drifts were followed by fast vergence responses in the opposite direction reestablishing binocular foveation of the small target (Fig. 4). Inspection of the eye movements of this subject during vergence tracking when no background was present showed that small drifts in vergence away from the target occurred in this condition too. However, in this situation these drifts did not affect the vergence responses noticeably. Inspection of the eye movements, made by the other subjects while they tracked the vertical bar, did not show


Fig. 3. Frequency responses of ocular vergence averaged over five subjects for different amplitudes of target-vergence. The stimulus (a single bar) was fixated in the absence (closed symbols) or presence (open symbols) of a stationary background containing dots (see Fig. 1A). Vertical bars indicate $1 \mathrm{SD}$

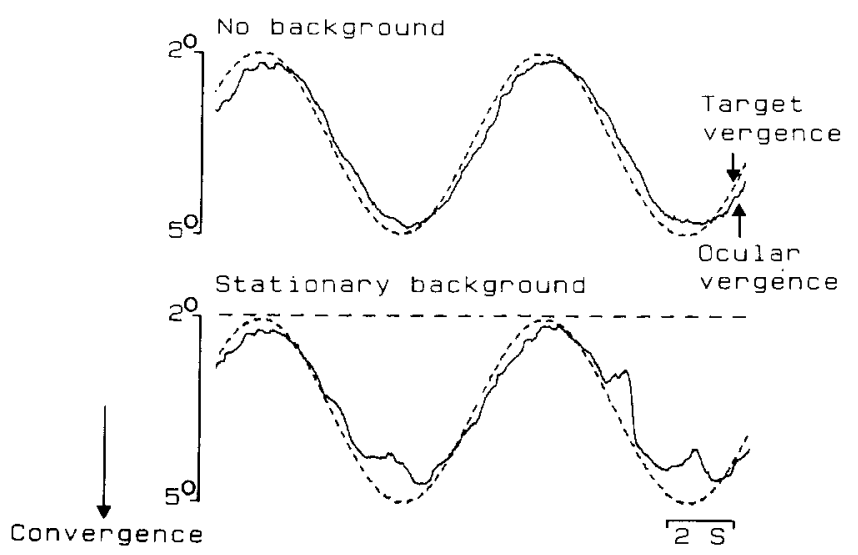

Fig. 4. Vergence responses of subject E.D. to sinusoidal changes in target-vergence of the stimulus (a single bar). The stimulus was viewed in the absence (top figure) or presence (bottom figure) of a stationary background containing dots (see Fig. 1A)

any noticeable drifts from the target. After removal of the data of the drifting subject from the data set, gains of ocular vergence did not differ significantly in the two stimulus conditions. 
The background influenced the perception of the small stereogram strongly, in contrast to the minor effects of the background on vergence. Without a background, the bar was fused continuously and moved only a little in depth during oscillations of low frequencies. During high frequency oscillations the bar was not fused and the two halfimages moved sideways. With a background, the bar was fused while the background was intermittently fused and not fused. The bar moved strongly in depth relative to the background with slow oscillations. During fast oscillations the bar was intermittently fused and not fused while the background was always fused. However, even in this case the background did not have any influence on vergence because the same vergence responses were measured when the background was absent.

\section{Experiment II: selective vergence tracking of stabilized targets}

In the preceding experiment it was established that in the presence of more than a single disparity, vergence was driven almost exclusively by the disparity of the selected target. From this result, however, it is not clear whether the retinal position of the selected target, located near the foveae during most of the time, was the decisive factor in the control of vergence, or whether the selection of a particular target as such made that target more powerful in driving vergence than all other potential targets. There are indications that a foveal stimulus drives vergence far more strongly than a peripheral stimulus does (Regan et al. 1986). In order to eliminate retinal position as a variable, two subjects were stimulated with retinal images which were electronically stabilized in the horizontal direction. Two targets were kept at opposite, parafoveal positions irrespective of any horizontal eye movements while the subject alternatingly selected one of them. The two subjects used in this experiment were experienced in viewing stabilized images and performed well in the previous experiments. Figures 5 and 6 show the results from this experiment for one subject. The results for the other subject were very similar to those presented here. Figure 5 shows movements of the right eye while it viewed the stimulus of Fig. 1B monocularly, while the left eye was covered. Attempted fixation of the bar in the right upper quadrant was accompanied by eye movements in the right direction. Attempted fixation of the bar in the left upper quadrant was associated with eye movements to the left. The eye movements were partially saccadic and partially smooth in nature. This mixture of saccadic and smooth eye movements is related to the parafoveal position of the attended bar. When the distance from the fovea is smaller than about $0.5^{\circ}$ the resulting eye movements will generally be smooth. With increasing distance from the fovea the eye movements will gradually contain more saccades.

The experimenter, who looked over the shoulder of the subject at the target on the screen, could infer the horizontal position of the subject's eye from the position of the target on the large screen. When the experimenter found that the target, and therefore also the eye of the subject, had rotated far enough in a specific direction, he asked the

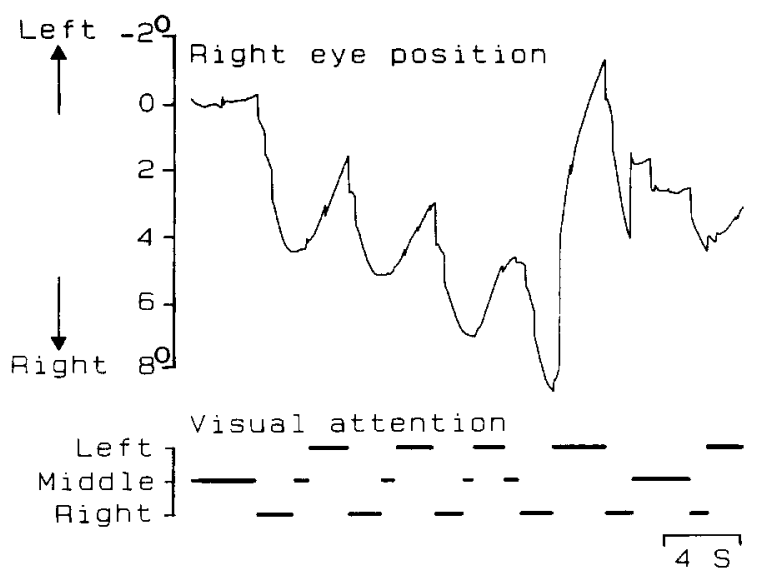

Fig. 5. Horizontal movements of the right eye while the subject viewed the stimulus (see Fig. 1B) monocularly with his right eye. The stimulus was horizontally stabilized on the retina. The subject alternatingly selected the left, middle or right vertical bar on command of the experimenter. The instructions recorded by the experimenter are indicated in the bottom of the figure

subject to attend to either the middle bar or the opposite small bar. The eye movement of the subject stopped after he was asked to change his attention to the middle bar, whereas the direction of his eye movement reversed when he was asked to attend to the other small bar (Fig. 5). The experimenter could effectuate that the subject made more or less oscillatory eye movements of a specific amplitude by giving him the instructions to displace the attention at the appropriate moments.

Eye movements during binocular viewing of the stimulus are shown in Fig. 6. The leftward and rightward movements of the subject's eyes followed the instructions of the experimenter similarly as in the monocular viewing condition. Attempted fixation of a bar flanking the vertical cross-bar on the left or right side was accompanied by eye movements in the corresponding direction. However, during binocular viewing the two eyes travelled with unequal speeds. This resulted in changes in ocular vergence. Attempted fixation of the right upper bar, which had a crossed disparity, was accompanied by convergent movements. By selecting the left upper bar, which had an uncrossed disparity, vergence movements immediately switched to the divergent direction. During attempted fixation of the bars in the lower quadrants, the relationship between conjugate and disjunctive eye movements was opposite. The eye movements contained more saccades during fixation of the upper part of the cross-bar (Fig. 6, upper panels) than during fixation of the lower part (Fig. 6, lower panels). This difference may be related to the fact that the distance of the small bars from the fovea were somewhat larger in the upper quadrants than in the lower quadrants (see Methods). During fixation of the lower part of the cross-bar, eye movements to the right were accompanied by divergent movements and left-ward eye movements by convergent movements. This means that convergent eye movements were associated with attempted fixation of those bars which had a crossed disparity, and divergent eye movements were associated with attempted 


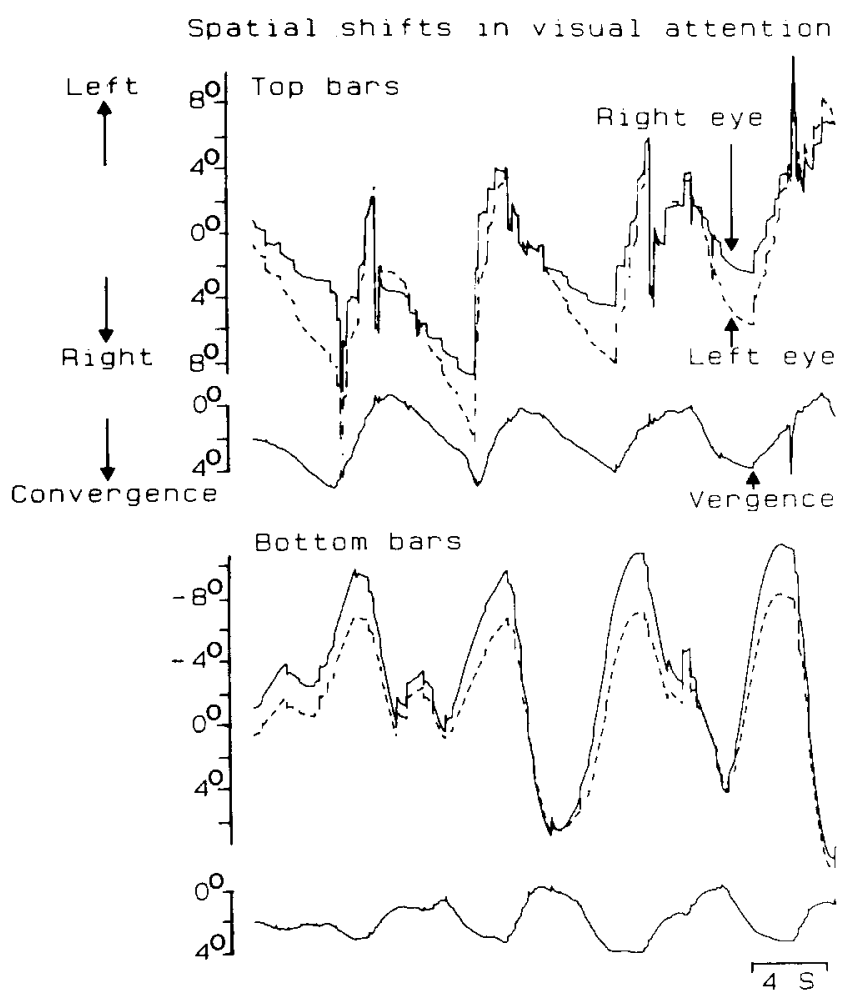

Fig. 6. Vergence together with horizontal movements of the two eyes while the subject viewed the stimulus (see Fig. 1B) binocularly. The stimuli were horizontally stabilized on the retinae. The subject alternatingly selected one of the vertical bars, flanking the vertical cross-bar in the upper quadrants (upper panel), or in the lower quadrants (lower panel)

fixation of the bars which had an uncrossed disparity. This implies that under normal viewing conditions the eye movements would have caused a reduction of the disparity of the selected target at the expense of increasing disparities of the non-selected targets. The opposite relationship between conjugate and disjunctive eye movements for targets in the upper and lower quadrants proved that vergence was truly driven by the disparity of the attended target, and was not an adventitious result of some unbalance of versional eye movements.

Vergence speeds ranged from $2.4^{\circ} / \mathrm{s}$ to $7.4^{\circ} / \mathrm{s}$ in the two subjects. The magnitudes of the vergence changes were about $4^{\circ}$ peak-to-peak and were essentially limited by the size of the concomitant conjugate eye movements. The experimenter took care that excursions of the eyes were kept within a range of about $15^{\circ}$ centered around the straight-ahead position in order to guarantee optimal image stabilization throughout the experiment.

\section{Discussion}

Self-generated eye movements with targets stationary on the retina have been reported before. Grüsser (1986) described smooth horizontal oscillating eye movements with a pair of horizontal point targets, stabilized at symmetrical positions with respect to the fovea, when the subject attended to each point target in turn in response to a periodical, auditory stimulus. Similarly, Kommerell and Täumer (1972) showed that a subject made a smooth eye movement with a foveally stabilized target in the direction in which he attempted to look when he attended to a blank zone close to the foveal target. The direction of the smooth eye movement reversed when the subject directed his attention to the other side of the foveal target. Kommerell and Täumer (1972) concluded that attention directed to an eccentric visual direction can be as valid as a visible target to elicit eye movements. The novelty of the present results is that the disparity information contained by the attended target dominated the vergence responses. Such vergence responses would have reduced disparity of the parafoveal, selected target if this had not been stabilized. Reduction of this disparity would have been achieved at the expense of increasing disparities of even foveal, but non-selected targets.

Delays as well as velocities of vergence responses, measured in the present experiments, agreed well to those found in the literature (Rashbass and Westheimer 1961; Erkelens 1987). This means that the dynamical properties of vergence were rather similar under different experimental conditions. The dynamics of vergence responses seems largely to be determined by disparity of the selected targets. In this respect the generation of vergence movements is different from the generation of limb or head movements. For instance, when a goal-directed hand movement is planned, the plan will include three elements: to where, when and how the movement will be made. How movements will be made, i.e. their speed and trajectory seem not to be under voluntary control when vergence movements are planned. This characteristic of control is not limited to vergence but seems to be a feature of eye movement control in general. For instance, it is not possible to make a saccade of a particular size at half the usual speed, or to make a smooth pursuit eye movement to a distant target. Eye movements are made in a stereotypical way as if features of the selected target, such as for instance its retinal position and disparity, are gated to the appropriate subsystems which generate the movements. Subsystems processing retinal disparity (Westheimer and Mitchell 1956), blur (Alpern and Ellen 1956) and change in size (Erkelens and Regan 1986) have been shown to be independent drivers of vergence. Selection of a target and timing of the response seem to be the variables by which eye movements can be controlled voluntarily. This view is supported by the observation that the quality of saccades (Becker and Fuchs 1969), of smooth pursuit (Steinbach and Held 1968), and of vergence (Erkelens et al. 1989b) made in darkness to an imagined target is poor.

The present results show that, even under reduced stimulus conditions, in which the depth of a target relative to other targets is signalled only by its retinal disparity, subjects can choose a target, fixate it binocularly and perceive it in binocular fusion, even in the presence of other targets with different disparities, which may be much larger and may even project onto the foveae. Humans have the ability to direct their foveae towards any target they choose, despite the competition of other targets, which 
may have features that are potentially much more powerful in driving vergence. This means that not the total disparity information of the stimulus is used for driving vergence, but only a particular part of it. Only a voluntarily selected part is used to serve as input for the subsystem processing disparity. In the Introduction we discussed experiments which showed that peripheral stimuli could induce vergence movements. The present results show that peripheral stimuli induces vergence movements when a subject attends to such a stimulus. The present results do not provide an explanation for the fact that vergence responses increase with the size and complexity of the stimulus. This feature of vergence may indicate that all available information is used for driving vergence unless it counteracts an appropriate response.

Acknowledgement. This research was partly supported by the Foundation for Medical Research MEDIGON, The Netherlands (grant no. 900-550-092).

\section{References}

Alpern M, Ellen P (1956) A quantitative analysis of the horizontal movements of the eyes in the experiments of Johannes Müller. II. Effect of variation in target separation. Am $J$ Ophthalmol 42: 296-303

Becker W, Fuchs AF (1969) Further properties of the human saccadic system: eye movements and correction saccades with and without fixation points. Vision Res 9: 1247-1258

Burian HM (1939) Fusional movements: the role of peripheral retinal stimuli. Arch Ophthalmol 21: 486-491

Collewijn H, Van der Mark F, Jansen TC (1975) Precise recording of human eye movements. Vision Res 15: 447-450

Erkelens CJ (1987) Adaptation of ocular vergence to stimulation with large disparities. Exp Brain Res 66: 507-516
Erkelens CJ (1988) Ocular vergence induced by changes in spatially selective visual attention. Perception 17: A8

Erkelens CJ, Regan D (1986) Human ocular vergence movements induced by changing size and disparity. J Physiol 379: 145-169

Erkelens CJ, Van der Steen J, Steinman RM, Collewijn H (1989a) Ocular vergence under natural conditions. I. Continuous changes of target distance along the median plane. Proc R Soc Lond B 236: 417-440

Erkelens CJ, Steinman RM, Collewijn H (1989b) Ocular vergence under natural conditions. II. Gaze shifts between real targets differing in distance and direction. Proc R Soc Lond B 236: 441-465

Grüsser OJ (1986). The effect of gaze motor signals and spatially directed attention on eye movements and visual perception. In: Freund HJ, Büttner U, Cohen B, Noth J (eds) Progress in brain research, Vol 64. Elsevier North Holland, Amsterdam, pp $391-404$

Hampton DR, Kertesz AE (1983) Fusional vergence response to local peripheral stimulation. J Opt Soc Am 73: 7-10

Kertesz AE (1981) Effect of stimulus size on fusion and vergence. J Opt Soc Am 71: 289-293

Kommerell G, Täumer R (1972) Investigation of the eye tracking system through stabilized retinal images. Bibl Ophthalmol 82: $288-297$

Rashbass C (1981) Reflexions on the control of vergence. In: Zuber BL (ed) Models of oculomotor behavior and control. CRC Press, Boca Raton, pp 139-148

Rashbass C, Westheimer G (1961) Disjunctive eye movements. J Physiol 159: 339-360

Regan D, Erkelens CJ, Collewijn H (1986) Visual field defects for vergence eye movements and for stereomotion perception. Invest Ophthalmol Vis Sci 27: 806-819

Robinson DA (1963) A method of measuring eye movements using a scleral search coil in a magnetic field. IEEE Trans Biomed Electron 10: 137-145

Steinbach MJ, Held R (1968) Eye tracking of observer-generated target movement. Science 161: 187-188

Westheimer G, Mitchell G (1956) Eye movement responses to convergent stimuli. Am Arch Ophthalmol 55: 848-856 